\title{
A Linear Algorithm for Optimal Orthogonal Drawings of Triconnected Cubic Plane Graphs
}

\author{
Md. Saidur Rahman, Shin-ichi Nakano and Takao Nishizeki * \\ Graduate School of Information Sciences \\ Tohoku University, Sendai 980-77, Japan.
}

\begin{abstract}
An orthogonal drawing of a plane graph $G$ is a drawing of $G$ in which each edge is drawn as a sequence of alternate horizontal and vertical line segments. In this paper we give a linear-time algorithm to find an orthogonal drawing of a given 3-connected cubic plane graph with the minimum number of bends. The best known algorithm takes time $O\left(n^{7 / 4} \sqrt{\log n}\right)$ for any plane graph of $n$ vertices.
\end{abstract}

\section{Introduction}

An orthogonal drawing of a plane graph $G$ is a drawing of $G$ with the given embedding in which each vertex is mapped to a point, each edge is drawn as a sequence of alternate horizontal and vertical line segments, and any two edges do not cross except at their common end. Orthogonal drawings have attracted much attention due to its numerous practical applications in circuit schematics, data flow diagrams, entity relationships diagrams, etc [ B96, K96, T87]. In particular, we wish to find an orthogonal drawing with the minimum number of bends. For the plane graph in Fig, 1(a), the orthogonal drawing in Fig. 1(b) has the minimum number of bends, that is, seven bends.

For a given planar graph $G$, if it is allowed to choose its planar embedding, then finding an orthogonal drawing of $G$ with the minimum number of bends is NP-complete [GT94]. However, Tamassia [T87] and Garg and Tamassia [GT96] presented algorithms which find an orthogonal drawing of a given plane graph $G$ with the minimum number of bends in $O\left(n^{2} \log n\right)$ and $O\left(n^{7 / 4} \sqrt{\log n}\right)$ time respectively unless it is allowed to choose its planar embedding, where $n$ is the number of vertices in $G$. They reduce the minimum-bend orthogonal drawing problem to a minimum cost flow problem. On the other hand, several lineartime algorithms are known for finding orthogonal drawings of plane graphs with a presumably small number of bends although they do not always find orthogonal drawings with the minimum number of bends [B96, K96].

In this paper we give a linear-time algorithm to find an orthogonal drawing of a 3-connected cubic plane graph with the minimum number of bends. To the best of our knowledge, our algorithm is the first linear-time algorithm to find an orthogonal drawing with the minimum number of bends for a fairly large class of graphs.

* E-mail: saidur@nishizeki.ecei.tohoku.ac.jp, \{nakano, nishi\}@ecei.tohoku.ac.jp 


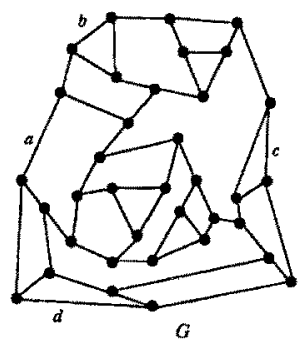

(a)

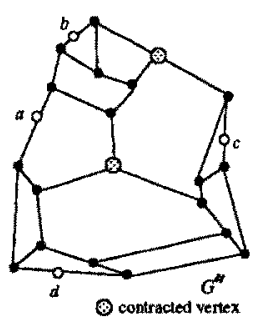

(d)

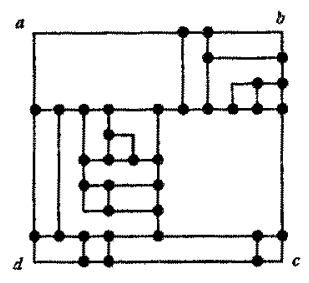

$G$

$(b)$

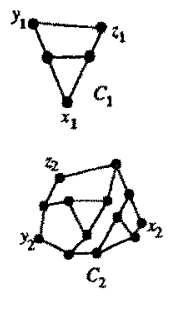

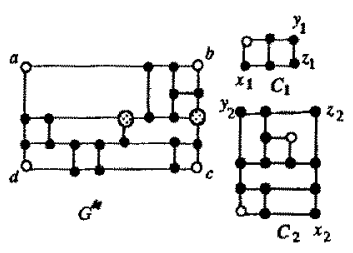

(e)

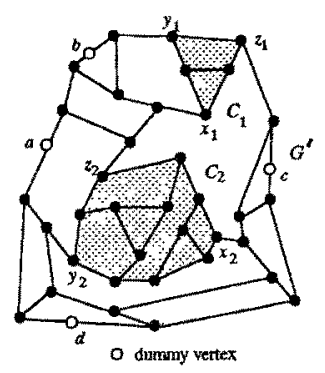

(c)

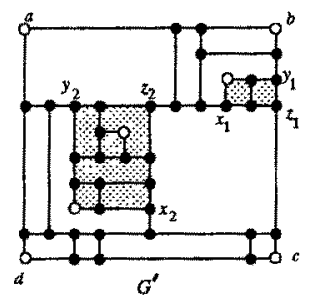

(f)

Fig. 1. A plane graph and its orthogonal drawing.

An orthogonal drawing in which there is no bend and each face is drawn as a rectangle is called a rectangular drawing. Linear-time algorithms have been known to find a rectangular drawing of a plane graph in which every vertex has degree three except four vertices of degree two on the outer boundary whenever such a graph has a rectangular drawing [KH94, RNN96]. The key idea of our algorithm is to reduce the orthogonal drawing problem to the rectangular drawing problem.

An outline of our algorithm is illustrated in Fig. 1. Given a plane graph as shown in Fig. 1(a), we first put four dummy vertices $a, b, c$ and $d$ of degree two on the outer boundary of $G$, and let $G^{\prime}$ be the resulting graph. The four dummy vertices are drawn by white circles in Fig. 1(c). We then contract each of some cycles $C_{1}, C_{2}, \cdots$ and their insides (shaded in Fig. 1(c)) into a single vertex as shown in Fig. $1(\mathrm{~d})$ so that the resulting graph $G^{\prime \prime}$ has a rectangular drawing as shown in Fig. 1(e). We also find orthogonal drawings of those cycles $C_{1}, C_{2}, \cdots$ and their insides recursively (see Figs. 1(d) and (e)). Patching the obtained drawings, we get an orthogonal drawing of $G^{\prime}$ as shown in Fig. 1(f). Replacing the dummy vertices $a, b, c$ and $d$ in the drawing of $G^{\prime}$ with bends, we finally obtain an orthogonal drawing of $G$ as shown in Fig. 1(b).

The rest of the paper is organized as follows. Section 2 gives some definitions and presents a known result. Section 3 presents an algorithm to find an orthogonal drawing in which the number of bends may not be the minimum but 
does not exceed the minimum number by more than four. Section 4 presents an algorithm to find an orthogonal drawing with the minimum number of bends, using the algorithm in Section 3. Finally Section 5 is a conclusion.

\section{Preliminaries}

In this section we give some definitions and present a known result.

Let $G$ be a connected graph with $n$ vertices and $m$ edges. We denote the set of vertices of $G$ by $V(G)$, and the set of edges of $G$ by $E(G)$. The degree of a vertex $v$ is the number of neighbors of $v$ in $G$. If every vertex of $G$ has degree three, then $G$ is called a cubic graph. The connectivity $\kappa(G)$ of a graph $G$ is the minimum number of vertices whose removal results in a disconnected graph or a single-vertex graph $K_{1}$. We say that $G$ is $k$-connected if $\kappa(G) \geq k$.

A graph is planar if it can be embedded in the plane so that no two edges intersect geometrically except at a vertex to which they are both incident. A plane graph is a planar graph with a fixed embedding. A plane graph divides the plane into connected regions called faces. We regard the contour of a face as a clockwise cycle formed by the edges on the boundary of the face. We denote the contour of the outer face of graph $G$ by $C_{o}(G)$.

For a simple cycle $C$ in a plane graph $G$, we denote by $G(C)$ the plane subgraph of $G$ inside $C$ (including $C$ ). We say that cycles $C_{1}$ and $C_{2}$ in a plane graph $G$ are independent if $G\left(C_{1}\right)$ and $G\left(C_{2}\right)$ have no common vertex. An edge which is incident to exactly one vertex of a simple cycle $C$ and located outside of $C$ is called a leg of the cycle $C$, and the vertex on $C$ to which the leg is incident is called a leg-vertex of $C$. A simple cycle with exactly $k$ legs is called a $k$-legged cycle.

An orthogonal drawing of a plane graph $G$ is a drawing of $G$ with the given embedding in which each vertex is mapped to a point, each edge is drawn as a sequence of alternate horizontal and vertical line segments, and any two edges do not cross except at their common end. A point where an edge changes its direction in a drawing is called a bend. We denote by $b(G)$ the minimum number of bends needed for an orthogonal drawing of $G$.

A rectangular drawing of a plane graph $G$ is a drawing of $G$ such that each edge is drawn as a horizontal or vertical line segment, and each face is drawn as a rectangle. Thus a rectangular drawing is an orthogonal drawing in which there is no bend and each face is drawn as a rectangle. The drawing of $G^{\prime \prime}$ in Fig. 1(e) is a rectangular drawing. The drawing of $G^{\prime}$ in Fig. 1(f) is not a rectangular drawing, but is an orthogonal drawing. The following result on rectangular drawings is known.

Lemma 1. Let $G$ be a connected plane graph such that all vertices have degree three except four vertices of degree two on $C_{o}(G)$. Then $G$ has a rectangular drawing if and only if $G$ has none of the following three types of simple cycles [T84]: (r1) 1-legged cycles, (r2) 2-legged cycles which contain at most one vertex of degree two, and (r3) 3-legged cycles which contain no vertex of degree two. 
Furthermore one can check in linear time whether $G$ satisfies the condition above, and if $G$ does then one can find a rectangular drawing of $G$ in linear time [RNN96].

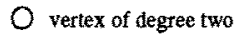

- vertex of degree three

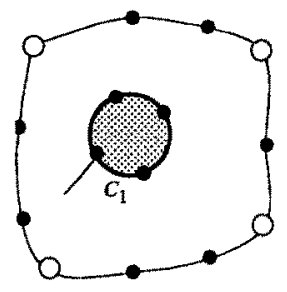

(a) 1-legged cycle

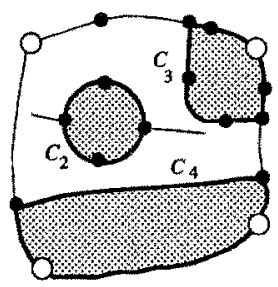

(b) 2-legged cycles

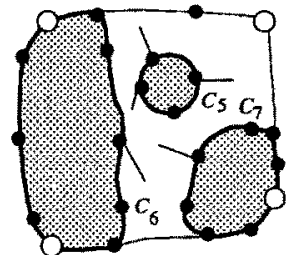

(c) 3-legged cycles

Fig. 2. Bad cycles $C_{1}, C_{2}, C_{3}$ and $C_{5}$, and non-bad cycles $C_{4}, C_{6}$ and $C_{7}$.

In a rectangular drawing of $G$, the four vertices of degree two are the four corners of the rectangle corresponding to $C_{o}(G)$. A cycle of type (r1), (r2) or (r3) is called a bad cycle. Figs. 2(a), (b) and (c) illustrate 1-legged, 2-legged and 3 -legged cycles, respectively. Cycles $C_{1}, C_{2}, C_{3}$ and $C_{5}$ are bad cycles. On the other hand, cycles $C_{4}, C_{6}$ and $C_{7}$ are not bad cycles; $C_{4}$ is a 2-legged cycle but contains two vertices of degree two, and $C_{6}$ and $C_{7}$ are 3 -legged cycles but contain one or two vertices of degree two.

Linear-time algorithms to find a rectangular drawing of a plane graph satisfying the condition in Lemma 1 have been obtained [KH94, RNN96]. Our orthogonal drawing algorithm uses the algorithm in [RNN96] which we call RectangularDraw in this paper.

\section{Orthogonal Drawing}

In this section we give a linear-time algorithm to find an orthogonal drawing of a 3-connected cubic plane graph $G$ with at most $b(G)+4$ bends. Thus there are at most four extra bends in a drawing produced by the algorithm.

Let $G$ be a 3 -connected cubic plane graph. Since $G$ is 3-connected, $G$ has no 1- or 2-legged cycle. A polygonal drawing of every cycle $C$ in $G$ has at least four convex corners, i.e., polygonal vertices of inner angle $90^{\circ}$, in any orthogonal drawing of $G$. Since $G$ is cubic, such a corner must be a bend if it is not a leg-vertex of $C$. Thus we have the following facts for any orthogonal drawing of $G$. 
Fact 2. At least four bends must appear on $C_{o}(G)$.

Fact 3. At least one bend must appear on each 3-legged cycle in $G$.

An outline of our algorithm is as follows.

Let $G^{\prime}$ be a graph obtained from $G$ by adding four dummy vertices $a, b, c$ and $d$ of degree two on $C_{o}(G)$ as follows. If $\left|E\left(C_{o}(G)\right)\right| \geq 4$, then add four dummy vertices on any four distinct edges on $C_{o}(G)$. If $\left|E\left(C_{o}(G)\right)\right|=3$, then add two dummy vertices on any two distinct edges on $C_{o}$ and two dummy vertices on the remaining edge.

If the resulting graph $G^{\prime}$ has luckily no bad cycle, then by Lemma $1 G^{\prime}$ has a rectangular drawing, in which the four dummy vertices become the corners of the rectangle corresponding to $C_{o}\left(G^{\prime}\right)$. From the rectangular drawing of $G^{\prime}$ one can immediately obtain an orthogonal drawing of $G$ with exactly four bends by replacing the four dummy vertices with bends at the corners. By Fact 2 the orthogonal drawing of $G$ has the minimum number of bends.

Thus we may assume that $G^{\prime}$ has a bad cycle. Since $G$ has no 1- or 2-legged cycle, every bad cycle in $G^{\prime}$ is a 3-legged cycle containing no vertex of degree two like $C_{5}$ in Fig. 2(c). Among all bad cycles of $G^{\prime}$, let $C_{1}, C_{2}, \cdots, C_{l}$ be the "maximal" ones, that is, those that are not located inside of any other bad cycle. Let $G^{\prime \prime}$ be the graph obtained from $G^{\prime}$ by contracting the inside $G^{\prime}\left(C_{i}\right)$ of each cycle $C_{i}, 1 \leq i \leq l$, into a single vertex. We find a rectangular drawing of $G^{\prime \prime}$, and recursively find a "suitable" orthogonal drawing of $G\left(C_{i}\right), 1 \leq i \leq l$, called a feasible drawing and defined later, and finally patch them to get an orthogonal drawing of $G$. (See Fig. 1.)

Before describing the algorithm in details, we analyze a hierarchical structure of 3-legged cycles in $G^{\prime}$. A 3-legged cycle $C$ in $G^{\prime}$ is called a descendant cycle of $C_{o}\left(G^{\prime}\right)$ if $C$ contains none of the four dummy vertices. We denote by $\mathcal{D}\left(G^{\prime}\right)$ the set of all descendant cycles of $C_{o}\left(G^{\prime}\right)$. A cycle $C$ in $\mathcal{D}\left(G^{\prime}\right)$ is called a child-cycle of $C_{o}\left(G^{\prime}\right)$ if $C$ is not located inside of any other cycle in $\mathcal{D}\left(G^{\prime}\right)$. Since $G$ is a 3-connected cubic plane graph, all child-cycles of $C_{o}\left(G^{\prime}\right)$ are independent each other. For two distinct cycles $C$ and $C^{*}$ in $\mathcal{D}\left(G^{\prime}\right)$, if $C^{*}$ is located in $G(C)$ but not located inside of any other 3-legged cycle in $G(C)$ except $C$, then $C^{*}$ is called a child-cycle of $C$. For any cycle $C \in \mathcal{D}\left(G^{\prime}\right)$, all child-cycles of $C$ are independent each other. Thus we get a hierarchical structure of cycles in $\mathcal{D}\left(G^{\prime}\right)$ represented by a "genealogical tree" $T_{g}$.

In Fig. 3(a) $\mathcal{D}\left(G^{\prime}\right)=\left\{C_{1}, C_{2}, C_{3}, C_{4}, C_{5}, C_{6}\right\}$. The 3-legged cycle $C_{7}$, indicated by a dashed line, is not in $\mathcal{D}\left(G^{\prime}\right)$ since it contains a dummy vertex $a$. Cycles $C_{1}, C_{2}, C_{3}$ and $C_{4}$ are the child-cycles of $C_{o}\left(G^{\prime}\right)$, and $C_{5}$ and $C_{6}$ are the child-cycles of $C_{4}$. The hierarchical structure of 3-legged cycles in $G^{\prime}$ in Fig. 3(a) is illustrated as a tree $T_{g}$ in Fig. 3(b).

Using a method similar to one in [RNN96], one can find such a hierarchical structure $T_{g}$ of 3-legged cycles in $G^{t}$ in linear time by traversing the contour of each face a constant number of times.

We assign the following information to each cycle in $\mathcal{D}\left(G^{\prime}\right)$ in a recursive manner from leafs to the root of $T_{g}$. Each cycle $C$ in $\mathcal{D}\left(G^{\prime}\right)$ is divided into 


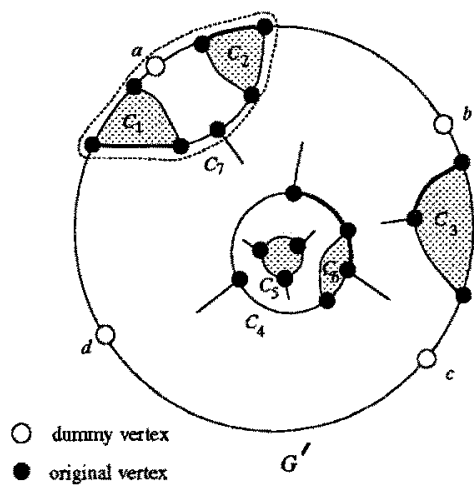

(a)

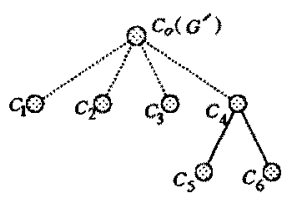

(b)

Fig. 3. (a) 3-legged cycles in $G^{\prime}$, and (b) a genealogical tree $T_{g}$.

three paths $P_{1}, P_{2}$ and $P_{3}$ by the three leg-vertices $x, y$ and $z$ of $C$. These three paths $P_{1}, P_{2}$ and $P_{3}$ are called the contour paths of $C$. Each contour path of $C$ is classified as a green path or a red path. In addition, each cycle $C$ in $\mathcal{D}\left(G^{\prime}\right)$ is assigned an integer $b c(C)$ called the bend-count of $C$. We will show later that $G(C)$ has an orthogonal drawing with $b c(C)$ bends and has no orthogonal drawing with fewer than $b c(C)$ bends, that is $b(G(C))=b c(C)$. Furthermore we will show that, for any green path of $C, G(C)$ has an orthogonal drawing with $b c(C)$ bends including a bend on the green path. On the other hand, for any red path of $C, G(C)$ does not have any orthogonal drawing with $b c(C)$ bends including a bend on the red path. We do these classification and assignment by the following bottom-up computation on the tree $T_{g}$, as follows.

Assume that we have already done the classification and the assignment for all child-cycles $C_{1}, C_{2}, \cdots, C_{l}$ of a cycle $C \in \mathcal{D}\left(G^{\prime}\right)$ and are going to do them for $C$. There are three cases.

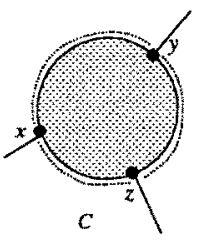

(a) Case 1

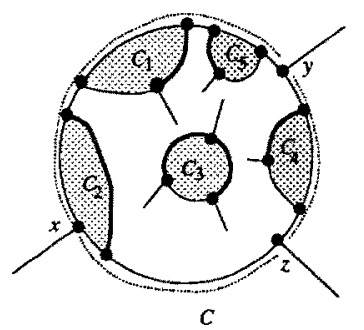

(b) Case 2

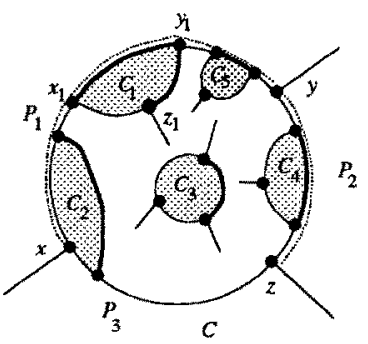

(c) Case 3

Fig. 4. Green paths. 
Case 1: $C$ has no child-cycle, that is, $l=0$ and $C$ is a leaf in $T_{g}$ (see Fig. 4(a)).

In this case, we classify all the three contour paths of $C$ as green paths, and set $b c(C)=1$. (By Fact 3 we need at least one bend.) In Fig. 4(a) green paths of $C$ are indicated by dotted lines.

Case 2: None of the child-cycles of $C$ has a green path on $C$.

(In Fig. 4(b) the child-cycles of $C$ are $C_{1}, C_{2}, \cdots, C_{5}$, and all green paths of them, drawn by thick lines, do not lie on $C$.) In this case, we classify all the three contour paths of $C$ as green paths, and set $b c(C)=1+\sum_{i=1}^{l} b c\left(C_{i}\right)$. Since none of $C_{1}, C_{2}, \cdots, C_{l}$ and their descendant cycles in $T_{g}$ has a green path on $C$, the orthogonal drawings of $G\left(C_{1}\right), G\left(C_{2}\right), \cdots, G\left(C_{l}\right)$ have no bend on $C$ and hence we need to introduce a new bend on $C$ in an orthogonal drawing of $G(C)$. In Fig. 4(b) the three green paths of $C$ are indicated by dotted lines.

Case 3: Otherwise (see Fig. 4(c)).

In this case at least one of the child-cycles $C_{1}, C_{2}, \cdots, C_{l}$, for example $C_{1}, C_{4}$ and $C_{5}$ in Fig. 4(c), has a green path on $C$. Classify a contour path $P_{i}, 1 \leq i \leq 3$, of $C$ as a green path if a child-cycle of $C$ has its green path on $P_{i}$. Otherwise, classify $P_{i}$ as a red path. Thus at least one of $P_{1}, P_{2}$ and $P_{3}$ is a green path. (In Fig. 4(c) $P_{1}$ and $P_{2}$ are green paths but $P_{3}$ is a red path.) We set $b c(C)=$ $\sum_{i=1}^{l} b c\left(C_{i}\right)$. (For a cycle $C_{j}$ having a green path on $C, G\left(C_{j}\right)$ has an orthogonal drawing with $b c\left(C_{j}\right)$ bends including a bend on the green path, and hence we need not to introduce any new bend on $C$.)

We have the following lemmas.

Lemma 4. At least one of the three contour paths of every 3-legged cycle in $\mathcal{D}\left(G^{\prime}\right)$ is a green path under the classification above.

Lemma 5. For every cycle $C$ in $\mathcal{D}\left(G^{\prime}\right), G(C)$ has at least bc $(C)$ vertex-disjoint 3-legged cycles in $G^{\prime}$ which contain no edge on red paths of $C$.

Proof. It is easy to prove the proposition by an induction based on $T_{g}$.

Lemma 6. For every cycle $C$ in $\mathcal{D}\left(G^{\prime}\right), b(G(C)) \geq b c(C)$.

Proof. By Fact 3 at least one bend must appear on each of the 3-legged cycles in $\mathcal{D}\left(G^{\prime}\right)$. By Lemma $5 G(C)$ has at least $b c(C)$ vertex-disjoint 3-legged cycles in $\mathcal{D}\left(G^{\prime}\right)$. Therefore any orthogonal drawing of $G(C)$ has at least $b c(C)$ bends, that is, $b(G(C)) \geq b c(C)$.

Conversely proving $b(G(C)) \leq b c(C)$, we have $b(G(C))=b c(C)$ for any cycle $C \in \mathcal{D}\left(G^{\prime}\right)$. Indeed we will prove a stronger claim later in Lemma 7 after introducing the following definition.

Let $x, y$ and $z$ be the three leg-vertices of $C$. One may assume that $x, y$ and $z$ appear on $C$ in clockwise order. For a green path $P$ with ends $x$ and $y$ on $C$, an orthogonal drawing of $G(C)$ is defined to be feasible for $P$ if the drawing satisfies the following properties $(\mathrm{p} 1)-(\mathrm{p} 3)$ : 
(p1) Exactly $b c(C)$ bends appear in the drawing of $G(C)$.

(p2) At least one bend appears on the green path $P$.

(p3) None of the the following six open halflines intersects the drawing of $G(C)$.

- the vertical open halfine with the upper end at $x$.

- the horizontal open halfline with the right end at $x$.

-- the vertical open halfline with the lower end at $y$.

- the horizontal open halfline with the left end at $y$.

- the vertical open halfline with the upper end at $z$.

- the horizontal open halfline with the left end at $z$.

It should be noted that the starting line segment of each leg of $C$ must lie on one of the six open halflines above in any orthogonal drawing of $G$ extended from an orthogonal drawing of $G(C)$ feasible for $P$.

Lemma 7. For any cycle $C$ in $\mathcal{D}\left(G^{\prime}\right)$ and any green path $P$ of $C, G(C)$ has an orthogonal drawing feasible for $P$.

Proof. We give an algorithm to find a feasible orthogonal drawing of $G(C)$ recursively, as follows. There are three cases.

Case 1: $C$ has no child-cycle.

In this case $b c(C)=1$. We insert, as a bend, a dummy vertex $t$ of degree two on an arbitrary edge on the green path $P$. Let $F$ be the resulting graph. Every vertex of $F$ has degree three except the three leg vertices $x, y$ and $z$, and the dummy vertex $t$. Furthermore $F$ has no bad cycle. Therefore by the algorithm Rectangular-Draw in [RNN96] one can find a rectangular drawing of $F$ with four corners on $x, y, z$ and $t$. The drawing of $F$ immediately yields an orthogonal drawing of $G(C)$ with exactly one bend at $t$. Thus the drawing satisfies (p1)-(p3), and hence is feasible for $P$.

Case 2: None of the child-cycles of $C$ has a green path on $C$.

Let $C_{1}, C_{2}, \cdots, C_{!}$be the child-cycles of $C$. First, we find an orthogonal drawing $D\left(G\left(C_{i}\right)\right)$ of $G\left(C_{i}\right)$ feasible for an arbitrary green path of $C_{i}$, for each $i=1,2, \cdots, l$, in a recursive manner.

Next, we construct a graph $F$ from $G(C)$ by contracting each $G\left(C_{i}\right), i=$ $1,2, \cdots, l$, to a single vertex $v_{i}$. We then construct a graph $H$ from $F$ by adding a dummy vertex $t$ on any of the edges of $P$ that remain in $F$. Thus there are exactly four vertices $x, y, z$ and $t$ of degree two on $C_{o}(H)$, and $H$ has no bad cycle. Therefore, by the algorithm Rectangular-Draw, we can find a rectangular drawing $D(H)$ of $H$ with four corners on $x, y, z$ and $t$. Fig. 5(a) illustrates $H$ for $C$ in Fig. 4(b).

Finally, patching the drawings $D\left(G\left(C_{1}\right)\right), D\left(G\left(C_{2}\right)\right), \cdots, D\left(G\left(C_{l}\right)\right)$ into $D(H)$, we can construct an orthogonal drawing of $G(C)$ with $b c(C)=1+\sum_{i=1}^{l} b c\left(C_{i}\right)$ bends (see Figs. 1 and 5). As illustrated in Fig. 6(b), there are twelve distinct embeddings of a contracted vertex $v_{i}$ and the three legs incident to $v_{i}$, depending on the directions of the three legs. For each of the twelve cases, we can replace a contracted vertex $v_{i}$ with a feasible orthogonal drawing of $G\left(C_{i}\right)$ or a rotated one shown in Fig. 6(c). Clearly $t$ is a bend on $P$ in the drawing of $G(C)$. Thus the drawing is feasible for $P$. 


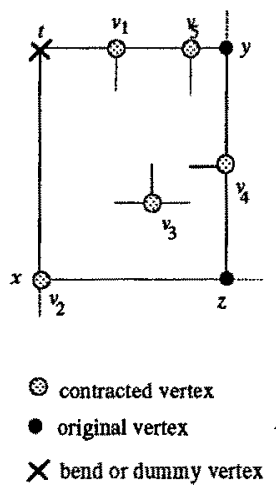

(a)

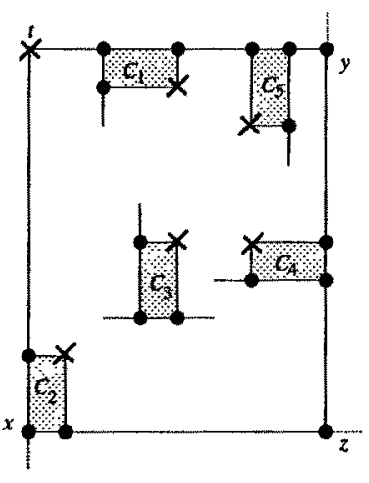

(b)

Fig. 5. (a) A rectangular drawing of $H$ and (b) a feasible orthogonal drawing of $G(C)$ for Case 2 .

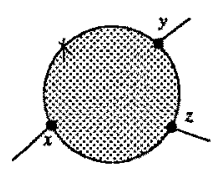

(a)

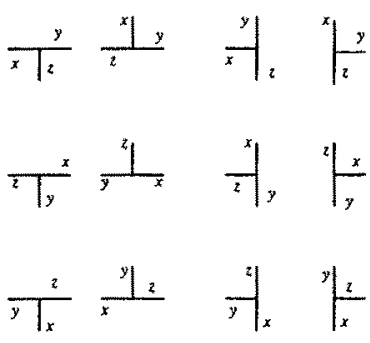

(b)
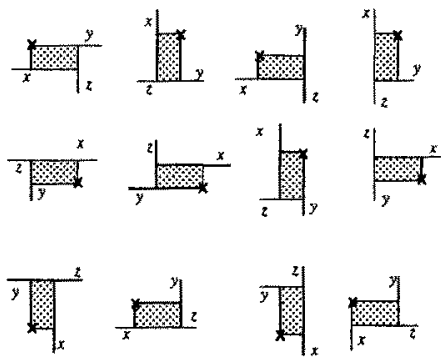

(c)

Fig. 6. (a) A 3-legged cycle, (b) twelve embeddings of a vertex $v_{i}$ and three legs incident to $v_{i}$, and (c) twelve feasible orthogonal drawings of $G\left(C_{i}\right)$ and rotated ones.

Case 3: Otherwise.

Let $C_{1}, C_{2}, \cdots, C_{l}$ be the child-cycles of $C$, where $l \geq 1$. In this case, a childcycle of $C$ has a green path on $C$. One may assume without loss of generality that $C_{1}$ has a green path $Q$ on the green path $P$ of $C$, that the three leg-vertices $x_{1}, y_{1}$ and $z_{1}$ of $C_{1}$ appear on $C_{1}$ clockwise in this order, and that $x_{1}$ and $y_{1}$ are the ends of $Q$. We first construct a graph $F$ from $G(C)$ by contracting each $G\left(C_{i}\right), 2 \leq i \leq l$, to a single vertex and by replacing $G\left(C_{1}\right)$ with a quadrangle $x_{1} t y_{1} z_{1}$, where $t$ is a dummy vertex of degree two. Thus $F$ has four vertices of degree two on $C_{o}(F)$, that is, $t$ and the three leg-vertices $x, y$ and $z$ of $C$. By the algorithm Rectangular-Draw we then find a rectangular drawing $D(F)$ of $F$ with four corners on $x, y, z$ and $t$, in which the contour $x_{1} t y_{1} z_{1}$ of a face is drawn as a rectangle. We next find an orthogonal drawing $D\left(G\left(C_{1}\right)\right)$ of $G\left(C_{1}\right)$ feasible for $Q$ and an orthogonal drawing $D\left(G\left(C_{i}\right)\right)$ of $G\left(C_{i}\right)$ feasible for an arbitrary green path of $C_{i}$ for each $i=2,3, \cdots, l$ in a recursive manner. Finally, patching the drawings $D\left(G\left(C_{1}\right)\right), D\left(G\left(C_{2}\right)\right), \cdots, D\left(G\left(C_{l}\right)\right)$ into $D(F)$, we can construct an 
orthogonal drawing of $G(C)$ feasible for $P$. Clearly $b c(C)=\sum_{i=1}^{l} b c\left(C_{i}\right)$ bends appear in the drawing of $G(C)$, and the bend $t$ appears on $Q$ and hence on $P$.

Lemmas 6 and 7 immediately imply the following Lemma 8.

Lemma 8. For every cycle $C$ in $\mathcal{D}\left(G^{\prime}\right), b(G(C))=b c(C)$.

The algorithm for finding a feasible orthogonal drawing of $G(C)$ described in the proof of Lemma 7 above will be called Feasible-Draw from now on. Rectangular-Draw takes linear time. As mentioned before, one can find $T_{g}$ in linear time by traversing every face boundary a constant number of times. Using $T_{g}$, one can easily compute $b c(C)$ and classify the three contour paths as green or red paths for all cycles $C \in \mathcal{D}\left(G^{\prime}\right)$ in linear time. Thus one can show that Feasible-Draw takes linear time. (The detail is omitted in this extended abstract.)

We are now ready to present our algorithm for orthogonal drawings of $G$.

\section{Algorithm Orthogonal-Draw $(G)$}

begin

1 add dummy vertices of degree two on $C_{o}(G)$ so that the resulting graph has four vertices of degree two on the contour of the outer face;

(if $C_{o}(G)$ has four or more edges, add four dummy vertices on four distinct edges, otherwise, add two dummy vertices on two distinct edges and two dummy vertices on the remaining edge.

2 let $G^{\prime}$ be the resulting graph;

3 let $C_{1}, C_{2} \cdots, C_{l}$ be the child-cycles of $C_{\circ}\left(G^{\prime}\right)$;

4 for each $i=1,2, \cdots, l$, find an orthogonal drawing $D\left(G\left(C_{i}\right)\right)$ of $G\left(C_{i}\right)$ feasible for an arbitrary green path of $C_{i}$ by algorithm Feasible-Draw;

5 let $G^{\prime \prime}$ be the graph derived from $G^{\prime}$ by contracting each $G\left(C_{i}\right), i=1,2, \cdots, l$, to a single vertex; $\left\{G^{\prime \prime}\right.$ has no bad cycle. $\}$

6 find a rectangular drawing of $D\left(G^{\prime \prime}\right)$ of $G^{\prime \prime}$ by algorithm Rectangular-Draw;

7 patch the drawings $D\left(G\left(C_{1}\right)\right), D\left(G\left(C_{2}\right)\right), \cdots, D\left(G\left(C_{l}\right)\right)$ into $D\left(G^{\prime \prime}\right)$ to get an orthogonal drawing of $G$

end.

We now have the following theorem.

Theorem 9. For any 3-connected cubic plane graph $G$, an orthogonal drawing of $G$ with at most $b(G)+4$ bends can be found in linear time.

Proof. (a) Number of bends.

There are two cases.

Case 1: $C_{o}(G)$ has no child-cycle.

In this case we have a drawing with exactly four bends. By Fact 2 it is a drawing with the minimum number of bends.

Case 2: Otherwise.

Let $C_{1}, C_{2}, \cdots, C_{l}$ be the child-cycles of $G^{\prime}$. Then we have an orthogonal drawing of $G$ with $4+\sum_{i=1}^{l} b c\left(C_{i}\right)$ bends. By Lemma $5 G$ has $\sum_{i=1}^{l} b c\left(C_{i}\right)$ vertexdisjoint 3-legged cycles. Therefore Fact 3 implies that $\sum_{i=1}^{l} b c\left(C_{i}\right) \leq b(G)$. Thus the number of bends in the derived drawing is at most $b(G)+4$. 
(b) Time complexity.

Orthogonal-Draw calls Rectangular-Draw for $G^{\prime \prime}$ and Feasible-Draw for $G\left(C_{i}\right)$, $1 \leq i \leq l$. Both Rectangular-Draw and Feasible-Draw take linear time. Furthermore cycles $C_{i}, 1 \leq i \leq l$, are independent each other. Therefore OrthogonalDraw takes linear time.

\section{Bend Minimization}

The algorithm Orthogonal-Draw in the preceding section finds an orthogonal drawing of a 3-connected cubic plane graph $G$ with at most $b(G)+4$ bends. In this section we give a linear-time algorithm to find an orthogonal drawing with the minimum number $b(G)$ of bends, using Orthogonal-Draw.

In Section 3 we have defined a child-cycle for the cycle $C_{o}\left(G^{\prime}\right)$ and for cycles in $\mathcal{D}\left(G^{\prime}\right)$. In this section we define a child-cycle for any 3 -legged cycle in $G$ as follows. For two distinct 3-legged cycles $C$ and $C^{*}$ in $G$, if $C^{*}$ is located in $G(C)$ but not located inside of any other 3-legged cycle in $G(C)$ except $C$, then $C^{*}$ is called a child-cycle of $C$. We also extend the definitions of a contour path, a green path, a red path and the bend-count $b c(C)$ for any 3-legged cycle $C$ in $G$.

Our idea is as follows. If $G$ has a 3-legged cycle which has a green path on $C_{o}(G)$, then we can save one of the four bends mentioned in Fact 2, because a bend on the green path can play a role of a bend in Fact 2 and also a role of a bend in Fact 3. We therefore want to find such 3-legged cycles as many as possible and up to four. We define a corner cycle to be a 3-legged cycle that has a green path on $C_{o}(G)$ but none of whose child-cycles has a green path on $C_{o}(G)$. If $G$ has independent corner cycles $C_{1}^{\prime}, C_{2}^{\prime}, \cdots, C_{k}^{\prime}, k \leq 4$, then we can save $k$ bends. We are now ready to give our algorithm to find an orthogonal drawing with the minimum number of bends.

Algorithm Minimum-Bend $(G)$

begin

1 find independent corner cycles $C_{1}^{\prime}, C_{2}^{\prime}, \cdots, C_{k}^{\prime}$ of $G$ as many as possible and up to four; $\{k \leq 4$. The detail of this step is omitted in this extended abstract. $\}$

2 let $P_{i}^{\prime}, 1 \leq i \leq k$, be a green path of $C_{i}^{\prime}$ on $C_{o}(G)$;

3 let $x_{i}, y_{i}$ and $z_{i}$ be the leg-vertices of $C_{i}^{\prime}$, and let the ends of $P_{i}^{\prime}$ be $x_{i}$ and $y_{i}$;

4 replace each subgraph $G\left(C_{i}^{\prime}\right), 1 \leq i \leq k$, in $G$ with a quadrangle $x_{i} t_{i} y_{i} z_{i}$ where $t_{i}$ is a dummy vertex of degree two;

5 let $G^{\prime}$ be the resulting graph;

6 Orthogonal-Draw $\left(G^{\prime}\right)$;

$7\left\{t_{1}, t_{2}, \cdots, t_{k}\right.$ are corners of the drawing of $C_{o}\left(G^{\prime}\right)$, and the quadrangle $x_{i} t_{i} y_{i} z_{i}$ is drawn as a rectangle for each $i, 1 \leq i \leq k$.

8 find an orthogonal drawing $D\left(G\left(C_{i}^{\prime}\right)\right)$ of $G\left(C_{i}^{\prime}\right)$ feasible for $P_{i}^{\prime}$ for each $i=1,2, \cdots, k$;

9 patch the drawings $D\left(G\left(C_{1}^{\prime}\right)\right), D\left(G\left(C_{2}^{\prime}\right)\right), \cdots, D\left(G\left(C_{k}^{\prime}\right)\right)$ into the drawing of $G^{\prime}$ to get an orthogonal drawing of $G$ end.

We now have the following theorem. 
Theorem 10. Algorithm Minimum-Bend produces an orthogonal drawing of a 3-connected cubic plane graph $G$ with the minimum number of bends in linear time.

\section{Conclusions}

In this paper we presented a linear-time algorithm to find an orthogonal drawing of a 3-connected cubic plane graph with the minimum number of bends. It is remained as a future work to find a linear-time algorithm for a larger class of graphs.

An orthogonal drawing is called an orthogonal grid drawing if all vertices and bends are located on integer grid points. Given an orthogonal drawing, one can find a corresponding (that means preserving the directions of edges) orthogonal grid drawing in linear time [T87]. Let $W$ be the width of a grid, that is the number of vertical lines in the grid minus one, and $H$ be the height of a grid. Let $n$ be the number of vertices, and let $m$ be the number of edges in a given graph. It is known that any orthogonal drawing using $b$ bends has a corresponding orthogonal grid drawing on a grid such that $W+H \leq b+2 n-m-2$ [B96]. It is also known that any 3 -connected cubic plane graph has an orthogonal grid drawing using at most $\frac{n}{3}+3$ bends on a grid such that $W \leq \frac{n}{2}$ and $H \leq \frac{n}{2}[\mathrm{~B} 96, \mathrm{~K} 96]$. By using our algorithm and the algorithm in [T87], one can find in linear time an orthogonal grid drawing of a 3-connected cubic plane graph with exactly $b(G)$ bends on a grid such that $W \leq \frac{n}{2}, H \leq \frac{n}{2}$ and $W+H \leq b(G)+2 n-m-2=b(G)+\frac{1}{2} n-2$.

\section{References}

[B96] T. C. Biedl, Optimal orthogonal drawings of triconnected plane graphs, Proc. of SWAT'96, LNCS 1097 (1996), pp. 333-344.

[GT94] A. Garg and R. Tamassia, On the computational complexity of upward and rectilinear planarity testing, Proc of Graph Drawing'94, LNCS 894 (1995), pp. 286-297.

[GT96] A. Garg and R. Tamassia, A new minimum cost flow algorithm with applications to graph drawing, Proc. of Graph Drawing'96, LNCS 1190 (1997), pp. 201-226.

[K96] G. Kant, Drawing planar graphs using the canonical ordering, Algorithmica 16 (1996), pp. 4-32.

[KH94] G. Kant and X. He, Two algorithms for finding rectangular duals of planar graphs, Proc. of WG'93, LNCS 790 (1994), pp. 396-410.

[RNN96] M. S. Rahman, S, Nakano and T. Nishizeki, Rectangular grid drawings of plane graphs, Proc. of COCOON'96, LNCS 1090 (1996), pp. 92-105. Also, Comp. Geom. Theo. Appl. (submitted).

[T87] R. Tamassia, On embedding a graph in the grid with the minimum number of bends, SIAM J. Comput. 16 (1987), pp. 421-444.

[T84] C. Thomassen, Plane representations of graphs, (Eds.) J.A. Bondy and U.S.R. Murty, Progress in Graph Theory, Academic Press Canada, (1984), pp. 43-69. 